\title{
Point-of-care ultrasonography in the diagnosis of hemorrhagic cholecystitis
}

\author{
Neil D. Long BMedSci BMBS, Justin S. Ahn MD, Gregory J. Monkewich MD, Daniel J. Kim MD
}

Cite as: CMAJ 2021 October 25;193:E1631-2. doi: 10.1503/cmaj.202394
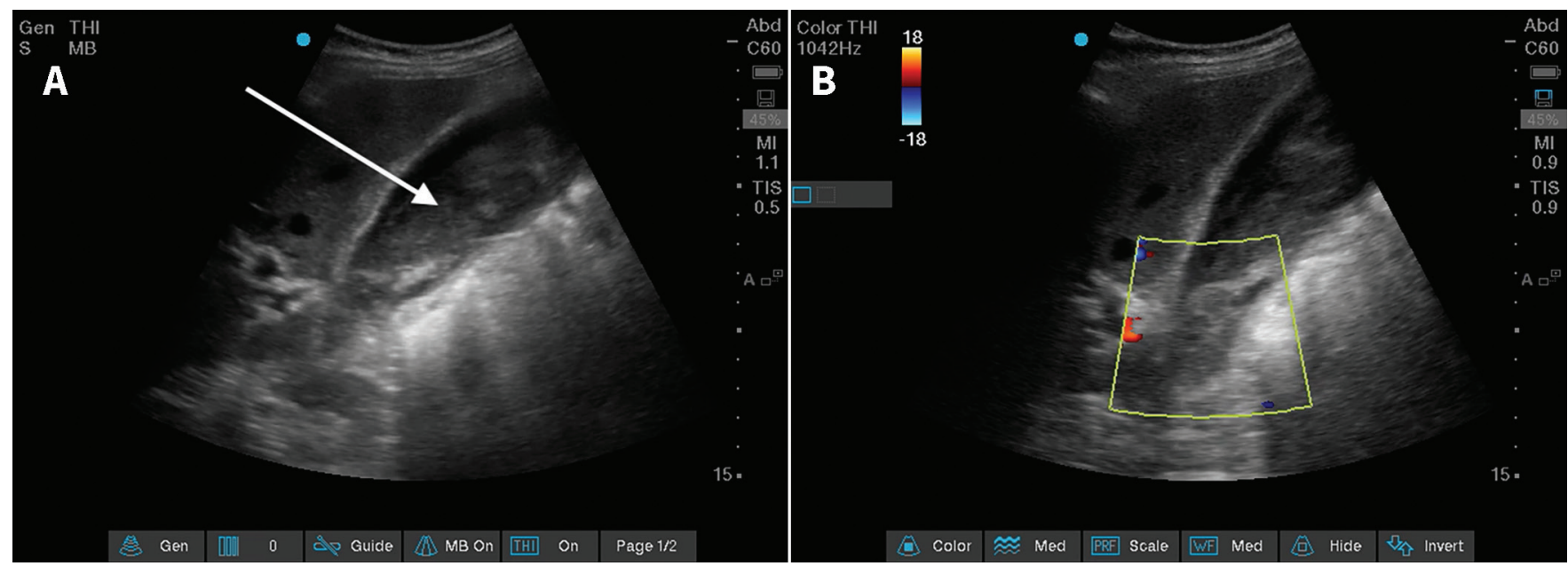

Figure 1: Ultrasonograms from an 81-year-old man with hemorrhagic cholecystitis. (A) Point-of-care ultrasonography shows the gallbladder lumen filled with mixed echogenic material (arrow), representing blood. (B) Colour Doppler ultrasonography of the gallbladder shows a lack of vascularity to the gallbladder wall and the echogenic material in the gallbladder lumen.

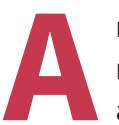

n 81-year-old man presented to the emergency department with a 1-day history of right upper quadrant abdominal pain, without fever or vomiting. Sixteen days before presentation, he had undergone coronary artery bypass surgery for an ST elevation myocardial infarction, and was prescribed clopidogrel and acetylsalicylic acid postoperatively. Physical examination showed right upper abdominal tenderness without guarding and a negative Murphy sign. Laboratory investigations showed elevated levels of white blood cells, bilirubin, aspartate transaminase, alanine transaminase and alkaline phosphate.

We performed point-of-care ultrasonography (POCUS), which showed heterogeneous echogenicity with layering that nearly filled the gallbladder lumen, without vascularity on colour Doppler ultrasonography. We did not observe any gallstones, wall thickening, pericholecystic fluid or sonographic Murphy sign (Figure 1 and Appendix 1 video, available at www.cmaj.ca/ lookup/doi/10.1503/cmaj.202394/tab-related-content). Ultrasonography performed in the radiology department confirmed POCUS findings.

Based on these findings, the patient underwent endoscopic retrograde cholangiopancreatography; sphincterotomy and sphincteroplasty yielded a large cast of bile-stained clot (Appendix 2, available at www.cmaj.ca/lookup/doi/10.1503/cmaj.202394/tab -related-content). As no predisposing structural or vascular abnormalities were detected, we diagnosed hemorrhagic cholecystitis secondary to the recent initiation of antiplatelet therapy. After the procedure, the patient's lab abnormalities and pain resolved, and he was discharged with consideration for an outpatient cholecystectomy once antiplatelet agents were discontinued.

Hemorrhagic cholecystitis is uncommon, but carries a substantial risk of morbidity and death, particularly if the gallbladder perforates. ${ }^{1}$ Hemorrhage within the gallbladder, with or without cholelithiasis, can be caused by trauma, biliary neoplasm, vascular abnormality, anticoagulation, antiplatelet drug use, bleeding diathesis or biliary parasites. ${ }^{2}$

Heterogeneous echogenicity filling the gallbladder lumen, with absence of flow on colour Doppler ultrasonography, are characteristics of hemorrhage within the gallbladder lumen. Pus can appear similarly heterogeneous, but biliary sludge is homogeneous on ultrasonography. Additional sonographic features of hemorrhagic cholecystitis include focal gallbladder wall thickening, intraluminal membranes, nonshadowing and nonmobile intraluminal echoes, or echogenic layering., ${ }^{1,3}$ In this case, POCUS facilitated management, expediting ultrasonography by the radiology department and surgical and gastroenterological consultation. 
Definitive treatment is cholecystectomy. For patients who cannot tolerate surgery, percutaneous cholecystostomy is a reasonable alternative. Endoscopic retrograde cholangiopancreatography is indicated if there is evidence of cholangitis or laboratory or radiologic signs of biliary obstruction from an organized clot, as in our case.

\section{References}

1. Jiang B, Bingmer K, Ammori J. Hemorrhagic cholecystitis. ACS Case Reviews in Surgery 2020;2:56-60.

2. Tavernaraki K, Sykara A, Tavernaraki E, et al. Massive intraperitoneal bleeding due to hemorrhagic cholecystitis and gallbladder rupture: CT findings. Abdom Imaging 2011;36:565-8.

3. Chinn DH, Miller EI, Piper N. Hemorrhagic cholecystitis. Sonographic appearance and clinical presentation. J Ultrasound Med 1987;6:313-7.

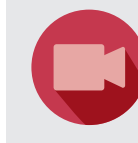

Videos of point-of-care ultrasonography and colour Doppler ultrasonography of an 81-year-old man with hemorrhagic cholecystitis are in Appendix 1, available at www.cmaj.ca/ lookup/doi/10.1503/cmaj.202394/tab-related-content
Clinical images are chosen because they are particularly intriguing, classic or dramatic. Submissions of clear, appropriately labelled highresolution images must be accompanied by a figure caption. A brief explanation (300 words maximum) of the educational importance of the images with minimal references is required. The patient's written consent for publication must be obtained before submission.
Competing interests: Daniel Kim is on the medical advisory board of Clarius Mobile Health. No other competing interests were declared.

This article has been peer reviewed.

The authors have obtained patient consent.

Affiliations: Department of Emergency Medicine (Long), Burnaby Hospital, Burnaby, BC; Department of Emergency Medicine (Long, Ahn, Kim), The University of British Columbia, Vancouver, BC; Department of Emergency Medicine, Royal Columbian Hospital (Ahn), New Westminster, BC; Department of Medicine (Monkewich), Burnaby Hospital, Burnaby, BC; Department of Emergency Medicine (Kim), Vancouver General Hospital, Vancouver, BC
Content licence: This is an Open Access article distributed in accordance with the terms of the Creative Commons Attribution (CC BY-NC-ND 4.0) licence, which permits use, distribution and reproduction in any medium, provided that the original publication is properly cited, the use is noncommercial (i.e., research or educational use), and no modifications or adaptations are made. See: https://creativecommons.org/licenses/ by-nc-nd/4.0/

Acknowledgements: The authors thank Dr. Gurp Johal and Dr. Jeremy White for reviewing this manuscript for accuracy from a general surgery and radiology perspective, respectively.

Correspondence to: Neil Long, expeditiondoctorlong@gmail.com 